\title{
A NOVEL PRESSURE BALANCED MICROFLUIDIC VALVE
}

\author{
J. M. Quero, A. Luque, L. G. Franquelo \\ Dpto. Ingeniería Electrónica \\ Universidad de Sevilla \\ Av. Camino de los Descubrimientos, s/n 41092 Sevilla, Spain \\ quero@esi.us.es
}

\begin{abstract}
A microfluidic valve capable of driving fluids under high (up to $10 \mathrm{~atm}$ ) pressure is presented. The valve actuation is based in a principle of pressure compensation by means of an air chamber that supports a moving membrane. The air will balance the fluid pressure, and then an electrostatic actuator can open the valve. This valve is designed with valve arrays in mind. In such configurations, it is useful for analysis, biology and medicine applications.
\end{abstract}

\section{INTRODUCTION}

Microvalves are fundamental parts of any fluidic microsystem [1]. These systems are every day more often used in different fields, such as biology, medicine, pharmaceutics, etc. In micro analysis or mixing systems, for example, a larger efficiency can be achieved by increasing flow that the system can manage. The easiest way to increase the flow is by increasing the pressure difference applied to the fluid.

Here arises the need of a microvalve capable of operating in a fluid under high pressures. The vast majority of designs actually in use cannot afford the requirements of efficiency needed by fluidic systems. There has been many attempts to design such a valve with actuations based on thermal expansion of paraffin [2], or thermally driven phase changes [3]. In some cases, fluid pressure itself is used to balance the forces acting on the moving parts of the valve [4].

A similar approximation can be taken by using an external fluid to compensate for the pressure differences. This approach has the advantage of easing the design of the valve, at the cost of introducing a new control fluid in the system. Once the pressure is balanced, another actuation is needed to open or close the valve. Among the designs widely in use, those based in electrostatic, electromagnetic [5], or even combined [6] actuations are preferred, mainly because of their controllability and low power consumption.

\section{VALVE DESIGN}

The fundamental idea behind the design is that by combining an electrostatic actuation with a pressure compensation system based on pneumatic effects, a high pressure microvalve can be obtained. To achieve this, the design starts with a traditional [7] normally-closed membrane valve actuated by electrostatic force. This kind of valve consists on a membrane orthogonal to the inlet and outlet orifices and that avoids the fluid to pass from inlet to outlet. On the other side of the membrane, an electrode can exert a force on it and move the membrane, so that the working fluid can flow through the valve.

The main problem with this design is that if the coming fluid pressure is too high, it can displace the membrane even if no electrostatic force is being applied and then the valve cannot avoid the fluid flowing. In some cases, the combination of fluid drag and electrostatic force has been successfully used to construct valves in MEMS technology, like the one described in [8].

The proposed solution consists in placing a chamber between the two electrodes. If this chamber is filled with high pressure air, then the membrane will resist the inlet pressure and will stop the flow, as desired. Note that air pressure need not be as high as inlet pressure, as the areas where these pressures are applied are not the same. If air pressure is adequately chosen, the membrane is near the equilibrium point and the electrostatic force applied by the electrodes is sufficient to open the valve.

The valve shown in fig. 1 has a circular membrane of about $450 \mu \mathrm{m}$ diameter. If the membrane was too small (typically less than $200 \mu \mathrm{m}$ ), then it would present a small deflection and the liquid flow will suffer from many losses. Separation between the electrodes is $5 \mu \mathrm{m}$ so that a high electrostatic force can be achieved and there is no need to be very precise in the air pressure in the chamber.

As shown in fig. 1, it is convenient that inlet orifice be centered with the membrane to obtain a better performance and to avoid irregular deflections that could lead to mechanical fracture of the membrane. 


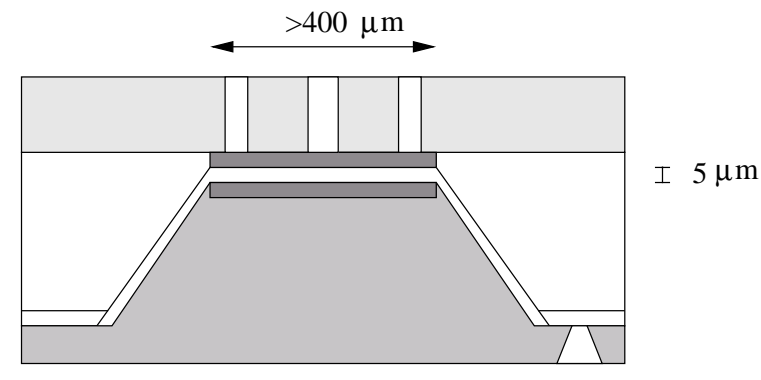

Fig. 1. Valve schematic

\section{VALVE ARRAY}

In applications such as mixing of fluids, a valve design capable of build arrays would be highly appreciated. Such a design must comply with two conditions: all valves must have the same design, and every valve must be controllable independently of the others.

These requirements can be fulfilled with the proposed design. The fundamental idea is that the air chamber can be shared between multiple valves in an array. Then, the same pressure is applied to all membranes, after a period of time needed to settle the pressure. In this case some of the membranes can be opened selectively by applying voltage to their electrodes.

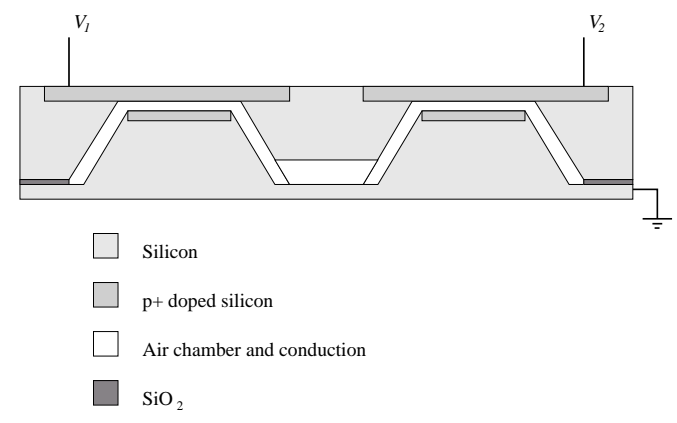

Fig. 2. Sample array of two valves

The main problem we are facing with when designing microvalve arrays is the routing of both the electric and the fluidic circuits.

In the electrical field, a practical solution consists in connecting all valve bases to a common ground, and arrange electrical connections that will drive the membranes to a high voltage with respect to this ground, and thus opening selectively the desired valves. The connection is carried at the $\mathrm{p}+$ doped areas showed in dark gray in fig. 2 .

The fluidic routing also presents important challenges. A typical application of the described valve may consist in mixing a variety of fluids in a specified order and in a specified ratio. In such applications, there would exist a number of fluid repositories that would fed the channels leading to the array of valves. Then the valves will open selectively for a period of time, calculated to allow the desired fluid amount to flow into the outlet channel and then into a collector.

If precision in amount of fluid deposited is important (which will often be the case), it is fundamental that fluidic losses will be equal along all channels that lead to the collector. This way, it is assured that the same opening time and same inlet pressure will lead to a an equal amount of fluid.

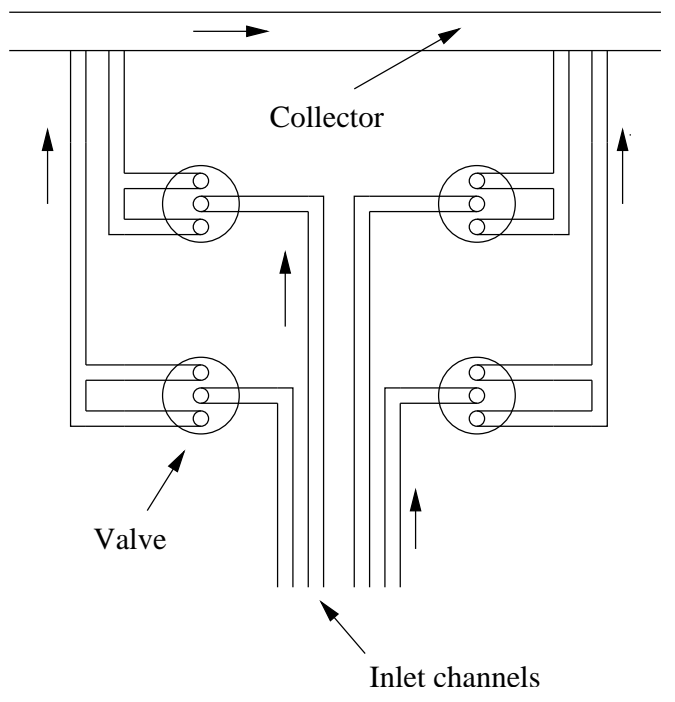

Fig. 3. Fluidic routing of four valves

With this approach, all valves can be built by repeating the same pattern along the mask, and routing appropriately the fluidic and electrical paths between them.

\section{OPERATION VARIABLES AND NUMERICAL VALUES}

When valve is closed, the only forces acting on the membrane are those caused by air pressure, and by the fluid in the inlet orifice. The valve is designed to support inlet pressures till $10 \mathrm{~atm}$ and remain closed. To assure that the membrane will not move, the following equilibrium condition must be fullfilled

$$
\pi r_{i}^{2} p_{i}=\pi r_{a}^{2} p_{a}
$$

where $p_{i}$ and $p_{a}$ are inlet fluid and air pressures, and $r_{i}$ and $r_{a}$ are the radii of inlet orifice and membrane, respectively. We are assuming both the membrane and the inlet orifice have circular form. So we obtain the pressure needed to keep valve closed as

$$
p_{a}=\left(\frac{r_{i}}{r_{a}}\right)^{2} p_{i}
$$


If we assume $r_{i}=60 \mu \mathrm{m}$ and $r_{a}=200 \mu \mathrm{m}$, we can use an air pressure as low as $0.09 p_{i}$, that is, $0.9 \mathrm{~atm}$. In fact, both pressures are not applied to the same area, and the inlet pressure acts over a more sensitive part of the membrane. Due to this fact, a somewhat greater air pressure must be used for security reasons. Care must be taken in not apply a too large pressure, because in that case the valve would not open with the electrostatic actuation. But another factor to be taken into account when choosing air pressure is the need to close the valve. When there is fluid circulating from the inlet to the outlet orifice over the membrane, its pressured is applied over the entire surface of the membrane, and thus if air pressure is too low, the valve would never close, even if electrostatic force is suppressed.

Electrostatic force applied to the membrane can be seen as a negative pressure acting on the bottom side of it. This equivalent pressure has a value of

$$
p_{e}=\frac{\varepsilon V^{2}}{2 g^{2}}
$$

where $V$ is the applied voltage, $g$ is the gap between electrodes and $\varepsilon$ is the dielectric constant of air between electrodes. For a voltage of $V_{g}=300 \mathrm{~V}$, we have an equivalent pressure of $0.16 \mathrm{~atm}$.

When an small electrostatic force that is not capable of driving the membrane to its open state is applied, there is actually a small deflection. This deflection always occurs in the central part of the membrane, where fluid is waiting to come into the valve. So the deflection allows the fluid to occupy more surface over the membrane, and thus to apply more force on it due to its pressure. This force leads to another increase of deflection, and so the effect has a positive feedback and will fully open the valve. It follows that it is not necessary to apply an equivalent electrostatic pressure of the same value as air pressure. Instead, we can open the valve with a equivalent pressure two orders of magnitude below the air pressure.

It has been shown that this valve has two equilibrium states: open and close. So, in its operation, it is bistable. In most applications, this is the only needed behaviour, but sometimes it is necessary to be capable of providing a required exact flow, maybe even adjusting it when there is some feedback from the fluidic system. In those cases, some control strategy can be implemented, being Pulse Width Modulation (PWM) the simplest of all.

\section{SIMULATION RESULTS}

Mechanical behaviour of the valve has been simulated by finite elements analysis using ANSYS. A model of a circular membrane was created, and correspondent boundary conditions applied. The forces acting on the membrane are air pressure, electrostatic force, and fluid pressure. The analysis also includes the limit on deflection caused by the lower electrode.

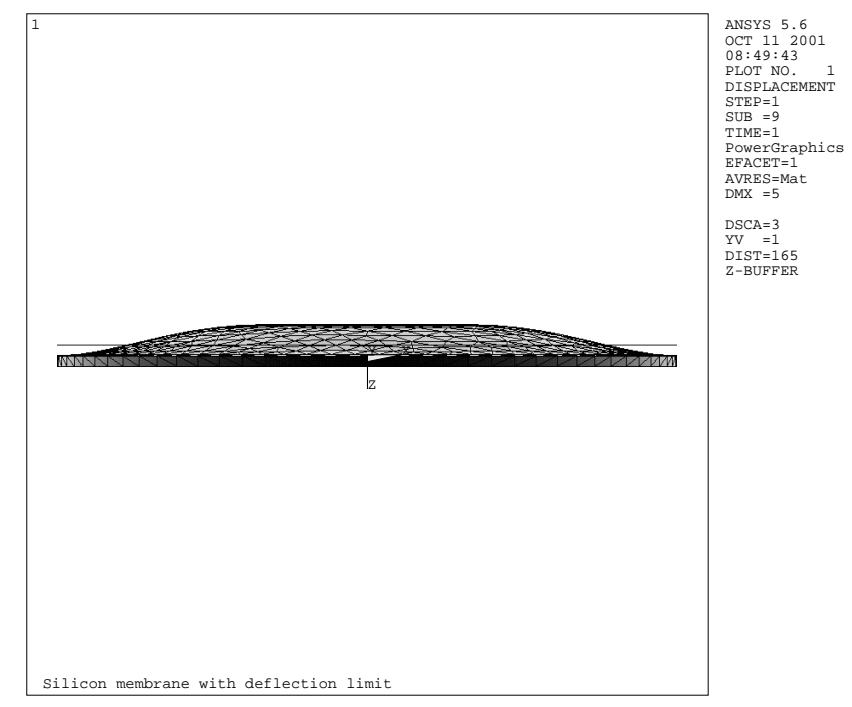

Fig. 4. Deformed shape of the membrane, with a limit on the deflection

In fig. 4 the deformed membrane is shown. The alignment of this figure is inverted with respect of the rest of figures in the document. The fluid is flowing in the lower side of the membrane, and the attracting electrode is located on the upper side. It can be seen that deformed shape is sensibly different from what we would obtain if we allowed a free deflection of the membrane.

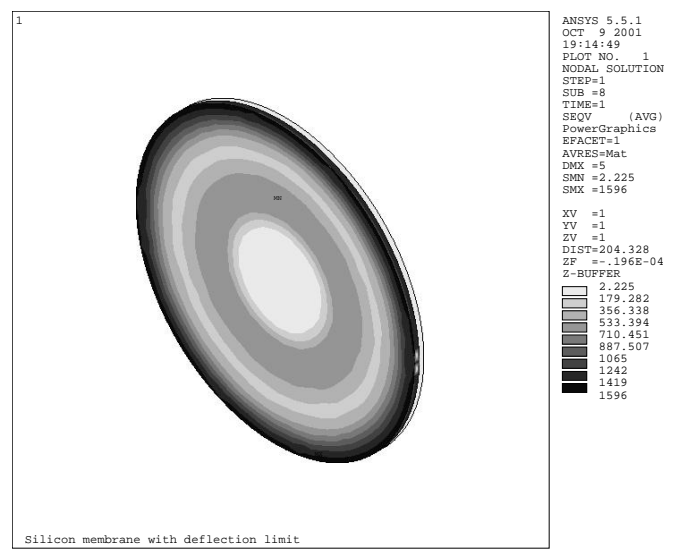

Fig. 5. Estimated stress on the membrane, with a limit on the deflection

This is better shown in fig. 5. This figure presents the stress in the membrane. The represented value is the equivalent Von Mises stress, useful for calculating possible plastic 
deformations and, more important, possible cracks in silicon. Like in fig. 4, the results are different from those obtained in a free deflection. When a solid limit is added, like in this case, the probability of silicon fracture is a 50.8 $\%$ of the original.

By simulation of different pressure differences applied to the valve, a response curve can be traced. Due to the laminarity of the flow, the relationship between pressure difference and flow obtained is almost lineal, as can be seen in fig: 6

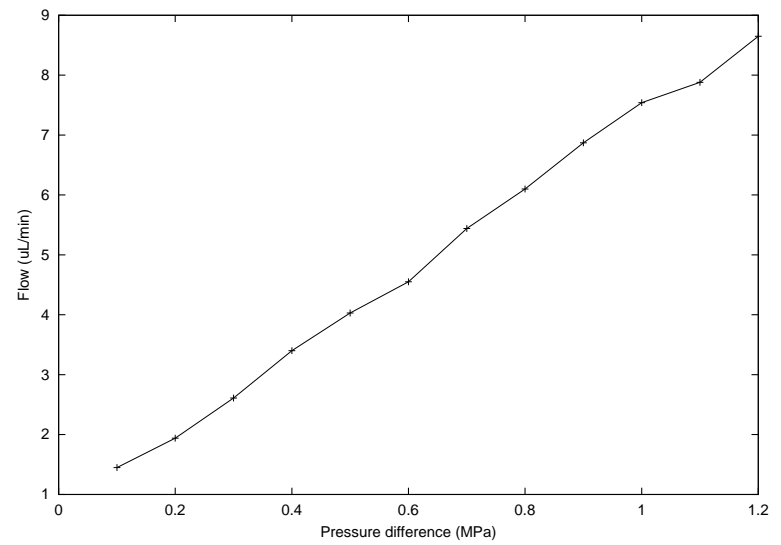

Fig. 6. Flow vs. pressure difference in fully opened valve

\section{FABRICATION PROCESS}

Both the membrane and the valve body are fabricated from p-type, (100) silicon wafers. In mambrane wafer, a silicon nitride is deposited using LPCVD. The selective etching of some areas of this film is accomplished by the use of a mask. Through the opneed hole, silicon is doped with boron, to obtain a $\mathrm{p}+$ area that will stop etching from the opposite side of the wafer. This etching is performed with $\mathrm{KOH}$, and will leave a membrane of approximately $5 \mu \mathrm{m}$. The exact thickness is determined by boron diffusion.

Similar steps are taken to build the valve body. Wafer is etched in some areas, to leave just a central elevated zone, as shown in fig. 1. After etching, solicon dixide layers are deposited in all exposed parts of the wafer.

The two wafers are joined together using anodic bonding. This process ensures that no gap will remain between them, so no air can escape the camera.

Another wafer is needed to contain the inlet and outlet orifices. This is normally made of glass. This layer can also be used to contain fluidic routing channels described in section 3 .

\section{CONCLUSIONS}

A new design for a microfluidic valve has been presented. As it has been said, its main advantage is the capability of operating under high pressures. Valve behavior has been simulated using finite elements analysis programs. Prototypes are under fabrication and will be available soon.

The advantage of using this valve in biological fields resides in its null effect on the working fluid. All electrical fields present in the valve are applied over air, and no voltage difference affects the fluid.

The valve here described is under patent P200102361.

\section{REFERENCES}

[1] S. Shoji and M. Esashi, "Microflow devices and systems," J. Micromech. Microeng., vol. 4, pp. 157-171, 1994.

[2] E. T. Carlen and C. H. Mastrangelo, "Paraffin actuated surface micromachined valve," in 13th IEEE Intl. Micro Electro Mechanical Systems Conference, 2000.

[3] P. L. Bergstrom, J. Ji, Y. Liu, M. Kaviani, and K. D. Wise, "Thermally driven phase-change microactuation," J. Microelectromech. Syst., vol. 4, pp. 10-17, Mar 1995.

[4] M. A. Huff, J. R. Gilbert, and M. A. Schmidt, "Flow characteristics of a pressure-balanced microvalve," in Tech. Dig. of Transducers'93, 1993, pp. 98-101.

[5] Melvin Khoo and Chang Liu, "A novel micromachined magnetic membrane microfluid pump," in 22nd Annual International Conference of the IEEE Engineering in Medicine and Biology Society, 2000.

[6] D. Bosch, B. Heimhofer, G. Muck, H. Seidel, U. Thumer, and W. Welser, "A silicon microvalve with combined electromagnetic/electrostatic actuation," Sensors and actuators, vol. A37-38, pp. 684-692, 1992.

[7] Paul Galambos, William P. Eaton, Randy Shul, Christi Gober Wilson, Jeffry J. Sniegowski, Samuel L. Miller, and Daniel Gutierrez, "Surface micromachining microfluidics: design, fabrication, packaging, and characterization," Available at http://www.mdl.sandia.gov/Micromachine.

[8] Levent Yobas, Michael A. Duff, Frederick J. Lisy, and Dominique M. Durand, "A novel bulk-micromachined electrostatic microvalve with a curved-compliant structure applicable for a pneumatic tactile display," J. Microelectromech. Syst., vol. 10, pp. 187-196, 2001. 